---مجلة علوم الرافدين، المجلد 27، العدد 4 /عدد خاص بالمؤتمر العلمي الثالث لعلوم الحياة ، ص 203-211، 2018---

$$
\begin{aligned}
& \text { عزل و تشخيص فطريات الهواء داخل وخارج ابنية مدارس مدينة تكريت } \\
& \text { نديم احمد رمضان } \\
& \text { بان بدران هوازن احمد عبد } \\
& \text { قسم علوم الحياة/ كلية العلوم/ جامعة الموصل لمصل } \\
& \text { قسم علوم الحياة/ كلية العلوم/ جامعة تكريت }
\end{aligned}
$$

$$
\text { (أستلم 2018/ }
$$

\title{
الملخص
}

تؤدي الفطريات دوراً هاماً في إصابة الإنسان بالعديد من الأمراض ومنها أمراض الحساسية. فقد وجد ان هناك العديد من الفطريات في هواء المدارس في مدينة تكريت خلال الموسم الاول (الخريفي) والموسم الثاني (الربيعي) تعود الى 24 جنسا، فضلا عن الفطريات العقيمة والخمائر • ووجد ان الفطر Cladosporium اكثر الفطريات حضوراً في هواء المدارس في نكريت (رياض الاطفال ومدارس الابتدائية للبنين وللبنات والثانوية للبنين والبنات) في الموسم الأول في الهواء الداخلي (39.13 و 30.76 و وكرئ

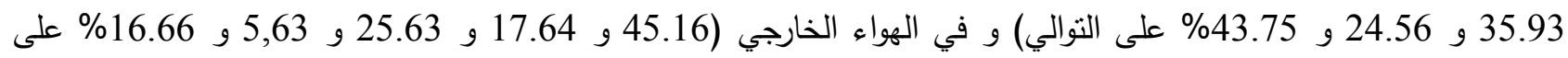
التوالي). كذللك تواجد بأعلى نكرار في الموسم الثاني في هواء رياض الاطفال والمدارس الابتدائية للبنات (61.29 و 16.9

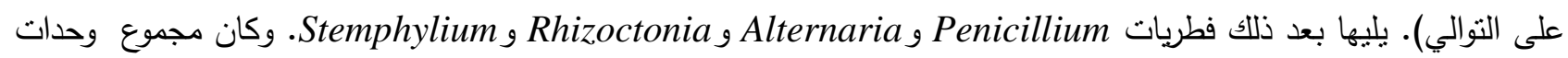
تكوين المستعرات (و.ت.م.) في الموسم الاول للفطر (58 ت.م.) وفي الموسم الثاني كان مجموع أعداد تكوين وحدات المستعمرات لفطر Cladosporium هو 124 و .ت. م. مو و فطر Alternaria الكلمات الدالة: فطريات الهواء، غبار المدارس، الفطر Cladosporium، فطريات هواء المدارس.

\section{Isolation and Diagnosis Indoor/ Outdoor Fungi of Schools in Tikrit City}

\author{
Ban Badran \\ Hawazin A. Abid \\ Department of Biology/College of Science / University of Tikrit \\ Nadeem A. Ramadan \\ Department of Biology/College of Science/ University of Mosul
}

\begin{abstract}
Fungi plays an important role in the infection of humans with many diseases, including allergies. It has been found that there are many fungi in schools air in the city of Tikrit during the first season (Autumn) and the second season (Spring) to 24 genera as well as sterile mycelium and yeast. Cladosporium was found to be the most common fungus in schools air in Tikrit (kindergartens and primary schools for boys and girls and secondary schools for boys and girls) in the first season in indoor air $(39.13,30.76,35.93,24.56$ and $43.75 \%$, respectively). And outdoor air (45.16, 17.64, 25.63, 5.63 and $16.66 \%$, respectively). There was also a repeat of the second season in the air of kindergartens and primary schools for girls (61.29 and 16.9\%, respectively) then the fungi Penicillium, Alternaria, Rhizoctonia and Stemphylium. The total number of colony formation units for Cladosporium $99 \mathrm{CFU}$ and followed by Penicillium (58CFU) in Autumn, in Spring Cladosporium (124 CFU) and Alternaria is $104 \mathrm{CFU}$.
\end{abstract}

Keywords: Air fungi, Schools dust, Cladosporium sp., Schooles dust fungi. 


\section{المقدمة}

اختصَّ الهه تعالى الفطريات بميزتين، بحيث تكون كائنات ضارة أو نافعة، لكنه من المؤسف أن كثبراً من العامة بظنون أنها

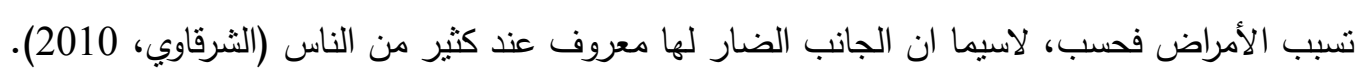
إن الفطريات Fungi هي كائنات حية "حقيقة النواة Eukaryotic" التي تكون مختلفة من ناحية السلوك والتركيب وكذلك

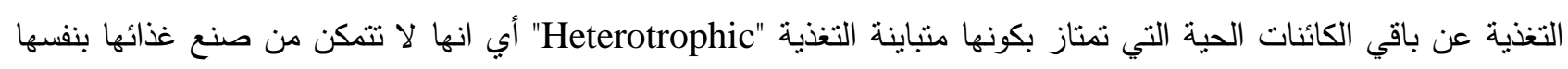

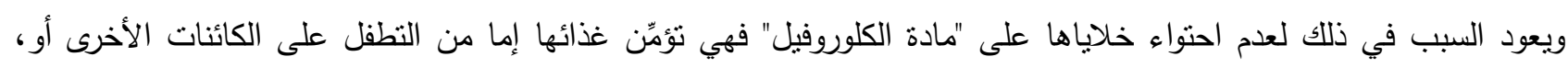

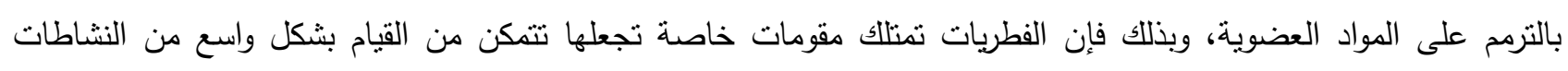
والفعاليات التي يمكن أن تدخل في مفاصل حياة الإنسان (نخيلان، 2011). اذ تتميز الفطريات بالقدرة على إنتاج السموم بأنواعها

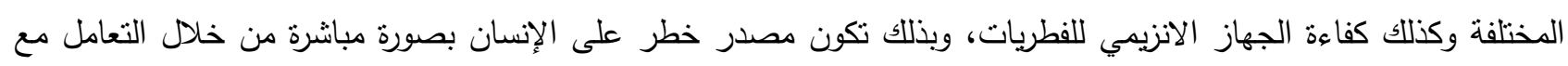

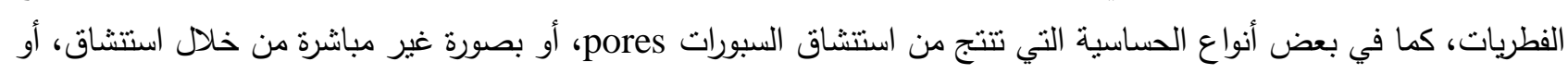

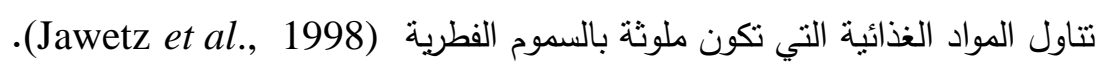

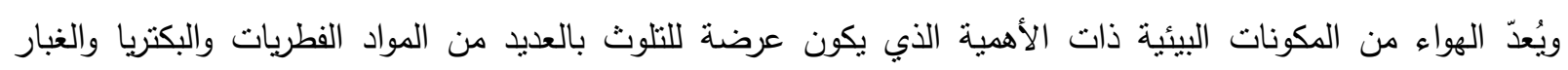

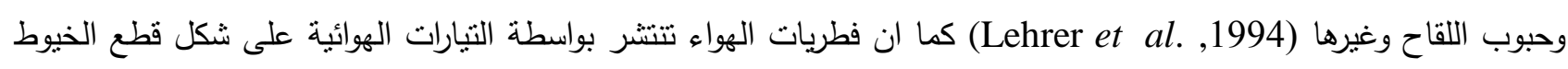
الفطرية والأبواغ، وتتمكن الكائنات الدقيقة وبضمنها الفطريات من أن تدخل الى المباني عن طريق دخول الهواء الخارجي وخلال

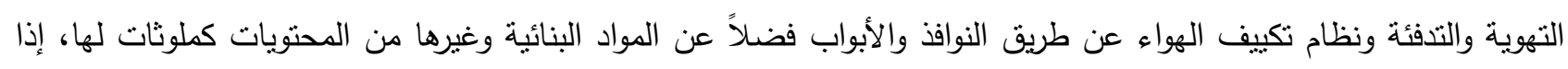
ارتفعت نسبة الرطوبة لفترة كافية (Shelton et al., 2002).

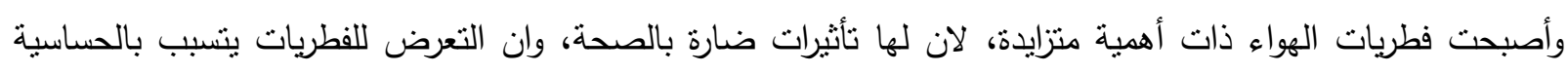
والتهيجات وغيرها من التأثيرات السامة، ومن خلال المعلومات التي يمكن الحُصول عليها من عينات فطريات الهواء في مساعدة الثقييم الطبي وكذلك تحديد طرائق علاجية وتقدير مدى المخاطر الصحية، وكذلك تقيد في قياس نوعية "الهواء الداخلي" بصورة منتابعة للحد من وقوع أي نوع من الأضرار (de Hoog et al., 1995 ; Hedayati et al. 2005 ; Shelton et al., 2002 ; Basilico et al., 2007). ورُصِدت ثلاث آليات للمرض الذي يمكن أن نسبيةُ الفطريات وهي : الحساسية والعدوى والنسمم الغذائي، أي انّ كلّ حالة من المرض ترتبط بنوع معين من الفطريات، وفي الآونة الأخيرة تكونت اختلافات كثثرة بنطاق واسع حول تأكيدات "غير مثبتنة علمياً" مفادها أن استتشاق فطريات الأماكن المغلقة يتسبب بأمراض معروفة ومحددة، غير أن هذه الأن الأمراض يمكن أن تتميز بالنقص في "التعريف التشريحي للمرض" وانعدام وجود الدليل الموضعي. كما أن الكثير من البحوث المنشورة منذ عام 1924 م التي عنيت بفكرة أن وجود الفطريات يمثل إحدى أسباب الإصابة بالربو الذي ينتج عن الحساسية وذلك بعد إثبات وجود الأجسام

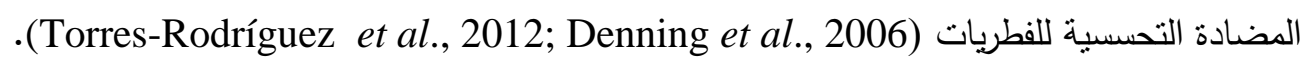
إن جميع أجزاء الفطر تكون مسببة للحساسية (الجراثيم الفطرية، والميسليوم وغيرها) ولها المقدرة على حث الأجسام المضادة، فضلا عن ان بعض السلالات الفطرية تضم على 40 أربعين فأكثر من "العوامل المسبية للحساسية عند الإنسان". وتتبير أغلب الدراسات العلمية التي تهتم بدراسة الفطريات الى أن عطية استتشاق أجزاء من الخيوط الفطرية أو جراثيم الفطريات تسبب عدوى الحساسية allergy و أمراض أخرى منها التسم، و يعتمد ذلك على نوع الفطر والفترة الزمنية للتعرض للهُ. وكذلك فأن الاستجابات للحساسية (الحساسية الأفية، الالتهاب الرئوي مفرط الحساسية، والربو) يمكن عَدُها من المشاكل الطبيعية والثنائعة على نطاق واسع وهي متعلقة باستنشاق الفطريات المتواجدة في الهواء (مشاط، 2013). 
تقع مدينة تكريت على الضفة اليمنى لنهر دجلة وعلى بعد 180 كيلومترا شمال مدينة بغدادو330 كيلومترا جنوب الموصل، وتميل بحافة شديدة الانحدار على نهر دجلة يتراوح ارتفاعها بين 45 - 50 م تقريباً، وهي منطقة شبه منموجة ترتفع عن سطح البحر 110. لذلك ارتأى البحث التحري عن الفطريات المنتشرة في هوائها داخل الأبنية (القاعات الدراسية)، وخارج الأبنية لرياض الأطفال والمدارس الابتدائية والإعدادية في مدينة تكريت. مواد و طرائق العمل

جمع العينات

Open plat method نمع فطريات الهواء داخل الأبنية المدرسية وخارجها لمدينة تكريت بطريقة الأطباق المفتوحة او Gravity settle plates) والحاوية على وسط البطاطا والدكستروز والاكار (PDA) Potato dextrose agar الثاني)، وشملت الدراسة رياض الأطفال ومدارس ابتدائية وثانوية وإعدادية للبنين و للبنات وبواقع مدرستين لكل مرحلة و 3 أطباق

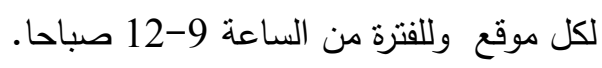
تحضير وسط البطاطا وإلدكستروز الصلب تم تحضير وسط البطاطا و الدكستروز PDA) Potato Dextrose agar) بإذابة 39 غم من المسحوق الجاهز من شركة (India-HiMedia) الوسط في دوارق مخروطية والتعقيم بالمقام. بعدها صُبَّ الوسط في الأطباق البلاستيكية حجم 50 ملم وتركت فيها لحين التصلب واستُخدمت فيما بعد.

عزل الفطريات عرضت الأطباق الحاوية على وسط (PDA) لهواء المدارس (رياض الأطفال والابتدائية والإعدادية) لمدة 10 دقائق بعدها

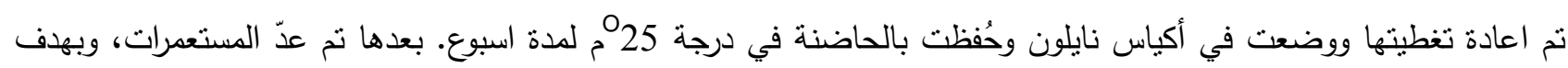

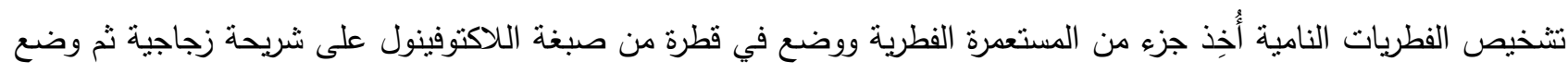
غطاء الثريحة وفحصت تحت المجهر ثنائي العدسة وشخصت الفطريات حسب المفاتيح التصنيفية الني وردت في المصادر الآتية: (Ellis,1971;Pitt and Hocking,1997;Watanabe, 2002).

\section{النتائج و المناقشة}

العزل من هواء رياض الأطفال تم الحصول على الفطريات من الهواء الغرف الداخلي والخارجي للغرف التي ينواجد فيها الأطفال وعُزل إحدى عشر جنسا

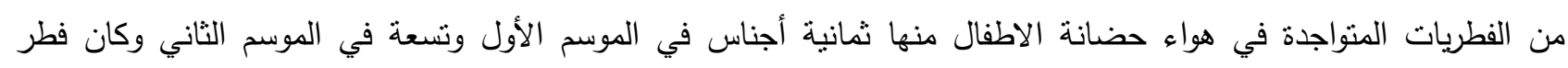
Cladiosporium

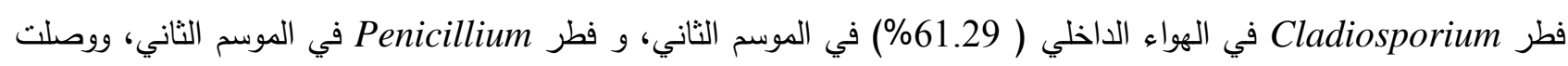

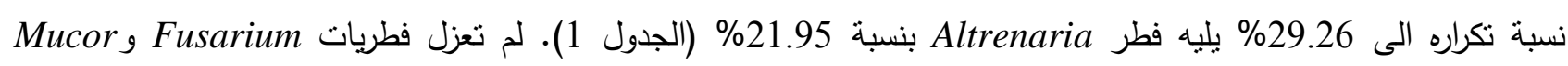
و Stemphylium والفطريات العقيمة في الموسم الثاني، فضلا عن عزل الخمائر في كلا الموسمين. وصلت أعداد الفطريات في الموسم الأول إلى 54 وحدة تكوينية مستعمرة، منها 23 في الهواء الداخلي و 31 
وحدة تكوينية مستعمرة في الهواء الخارجي، وفي الموسم الثاني كان أعداد وحدات تكوين المستعمرات في الهواء الداخلي والخارجي كثيرة إذ بلغت 72 منها 31 في الهواء الداخلي و 41 في الهواء الخارجيه وفي. وذكر (2012) Charles et al., احد فطر الفطريات المسببة للحساسية وأكثرها تواجدا في الهواء الداخلي والخارجي.

الجدول 1: الفطريات المعزولة من الهواء الداخلي والخارجي لرياض الاطفال في مدينة تكريت

\begin{tabular}{|c|c|c|c|c|c|c|c|c|c|c|}
\hline \multicolumn{5}{|c|}{ الموسم الثاني } & \multicolumn{5}{|c|}{ الموسم الاول } & \multirow[t]{3}{*}{ الفطريات } \\
\hline$\%$ & الهواء & $\%$ & الهواء & وحدة & $\%$ & الهواء & $\%$ & الهواء & وحدة & \\
\hline ل ل لتكرار & الخارجي & ل لتكرار & الداخلي & مستعمرين & ل ل لتكرار & الخارجي & ل لتنكرار & الاخلي & مستعمرين & \\
\hline 21.95 & 9 & 6.45 & 2 & 11 & 6.45 & 2 & 13.04 & 3 & 5 & Alternaria alternate \\
\hline 4.87 & 2 & 6.45 & 2 & 4 & 9.67 & 3 & 4.34 & 1 & 4 & Aspergillus spp \\
\hline 7.31 & 3 & 6.45 & 2 & 5 & 645 & 2 & 4.34 & 1 & 3 & Aureobasidium pullulans \\
\hline 0 & 0 & 61.29 & 19 & 19 & 45.16 & 14 & 39.13 & 9 & 23 & Cladosporium spp \\
\hline 4.87 & 2 & 0 & 0 & 2 & 0 & 0 & 8.69 & 2 & 2 & Drechslera spp \\
\hline 4.87 & 2 & 0 & 0 & 2 & 0 & 0 & 0 & 0 & 0 & Fusarium oxysporum \\
\hline 2.43 & 1 & 0 & 0 & 1 & 0 & 0 & 0 & 0 & 0 & Mucor spp. \\
\hline 29.26 & 12 & 9.67 & 3 & 15 & 6.45 & 2 & 13.04 & 3 & 5 & Pencillium spp \\
\hline 7.31 & 3 & 9.67 & 3 & 6 & 22.58 & 7 & 4.34 & 1 & 8 & Rhizoctoni solani \\
\hline 0 & 0 & 0 & 0 & 0 & 0 & 0 & 4.34 & 1 & 1 & Trichophyton spp \\
\hline 4.87 & 2 & 0 & 0 & 2 & 0 & 0 & 0 & 0 & 0 & Stmphylium spp. \\
\hline 0 & 0 & 0 & 0 & 0 & 3.22 & 1 & 0 & 0 & 1 & Steril mycelium \\
\hline 12.19 & 5 & 0 & 0 & 5 & 0 & 0 & 8.69 & 2 & 2 & Yeast \\
\hline 100 & 41 & 100 & 31 & 72 & 100 & 31 & 100 & 23 & 54 & 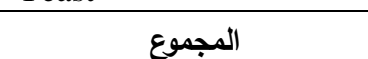 \\
\hline
\end{tabular}

\section{العزل من هواء المدارس الابتدائية}

وُجد ان هناك ستة عشر جنسا، فضلاً عن الفطريات العقيمة والخمائر تم عزلها في الموسمين من هواء المدارس الابتدائية

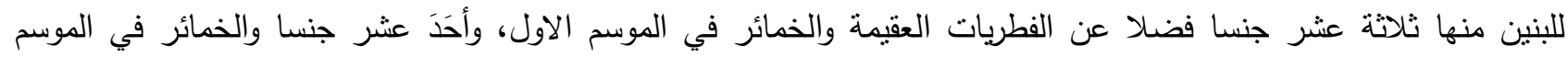

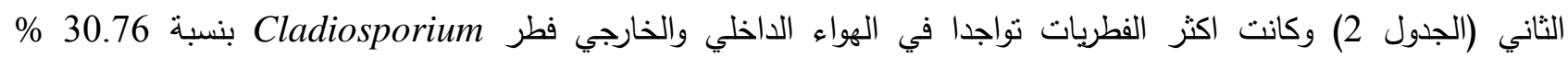
و17.64\% على التوالي. و في الموسم الثاني كان فطر Aureobasidium اكثر نواجداً في الهواء الداخلي بنسبة 22.22\% ، وفطر Alternaria بنسبة 35.29 \% في الهواء الخارجي. ووُجد ان اعداد تكوين المستعمرات في الموسم الأول اقل من الموسم

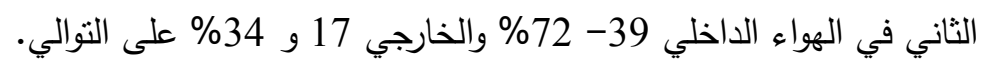
ونتائج (الجدول 3) نوضح ان الفطريات المعزولة من الهواء الداخلي و الخارجي تعود الى سبعة اجناس عزلت في الموسم

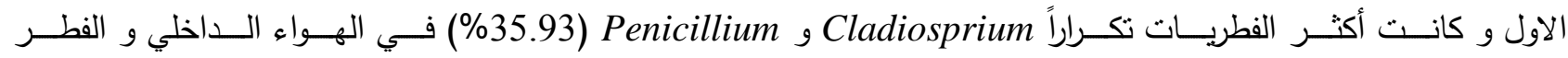

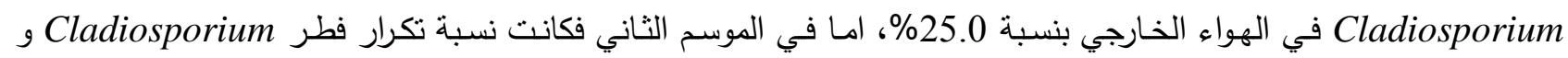
16.90 Rhizoctonia

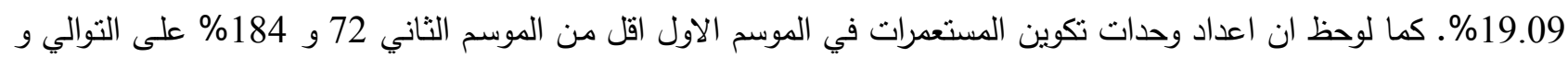

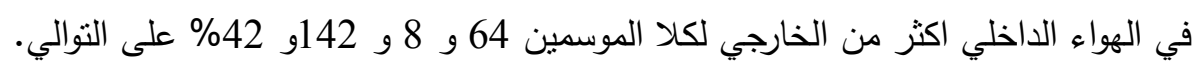


عزل و نتخيص فطريات الهواء.

الجدول 2: الفطريات المعزولة من الهواء الداخلي والخارجي لمدارس البنين الابتدائية في مدينة تكريت

\begin{tabular}{|c|c|c|c|c|c|c|c|c|c|c|}
\hline \multicolumn{5}{|c|}{ الموسم الثاني } & \multicolumn{5}{|c|}{ الموسم الاول } & \multirow[t]{4}{*}{ الفطريات } \\
\hline$\%$ & الهواء & $\%$ & الهواء & وحدة & $\%$ & الهواء & $\%$ & الهواء & وحدة & \\
\hline للتكرار & الخارجي & للتكرار & الداخلي & تكوين & للتكرار & الخارجي & للتكرار & الداخلي & تكوين & \\
\hline & & & & مستعمرة & & & & & مستعمرة & \\
\hline 35.29 & 12 & 13.88 & 10 & 22 & 5.88 & 1 & 7.69 & 3 & 4 & \\
\hline 0 & 0 & 0 & 0 & 0 & 0 & 0 & 2.56 & 1 & 1 & Aschochyta spp. \\
\hline 2.94 & 1 & 1.38 & 1 & 2 & 11.76 & 2 & 2.56 & 1 & 3 & Aspergillus spp \\
\hline 14.70 & 5 & 22.22 & 16 & 21 & 11.76 & 2 & 2.56 & 1 & 3 & Aureobasidium pullulans \\
\hline 11.76 & 4 & 15.27 & 11 & 15 & 17.64 & 3 & 30.76 & 12 & 15 & Cladosporium spp \\
\hline 0 & 0 & 1.38 & 1 & 1 & 0 & 0 & 2.56 & 1 & 1 & Drechslera spp \\
\hline 0 & 0 & 0 & 0 & 0 & 7.14 & 2 & 3.57 & 1 & 3 & Fusarium spp. \\
\hline 0 & 0 & 1.38 & 1 & 1 & 0 & 0 & 0 & 0 & 0 & Helminthesporium spp. \\
\hline 2.94 & 1 & 0 & 0 & 1 & 0 & 0 & 0 & 0 & 0 & Macrophmina phaseolina \\
\hline 0 & 0 & 0 & 0 & 0 & 0 & - & 3.57 & 1 & 1 & Mucor spp. \\
\hline 5.88 & 2 & 8.33 & 6 & 8 & 11.76 & 2 & 7.69 & 3 & 5 & Penicillium spp \\
\hline 14.70 & 5 & 9.72 & 7 & 12 & 0 & 0 & 2.56 & 1 & 1 & Rhizoctoni solani \\
\hline 0 & 0 & 0 & 0 & 0 & 0 & 0 & 2.56 & 1 & 1 & Rhizopus stolonifer \\
\hline 5.88 & 2 & 19.44 & 14 & 16 & 11.76 & 2 & 7.69 & 3 & 5 & Stemphylium herbarum \\
\hline 0 & 0 & 1.38 & 1 & 1 & 0 & 0 & 0 & 0 & 0 & Trichoderma spp \\
\hline 0 & 0 & 0 & 0 & 0 & 0 & 0 & 2.56 & 1 & 1 & Ulocladium spp. \\
\hline 0 & 0 & 0 & 0 & 0 & 11.76 & 2 & 7.69 & 3 & 5 & Steril mycelium \\
\hline 5.88 & 2 & 5.55 & 4 & 6 & 11.76 & 2 & 12.81 & 5 & 7 & Yeast \\
\hline 100 & 34 & 100 & 72 & 106 & 100 & 17 & 100 & 39 & 56 & المجموع \\
\hline
\end{tabular}

\section{العزل من هواء المدارس الإعدادية}

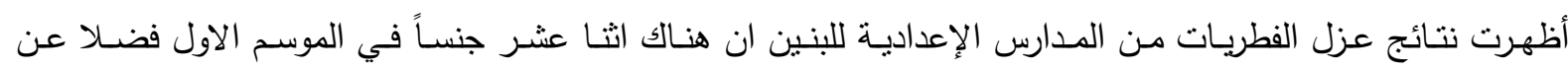
الفطريات العقيمة و الخمائر و ستة عشر جنسا فضلا عن الفطريات العقيمة و الخمائر في الموسم الثاني، وكانت اكثر الفطريات

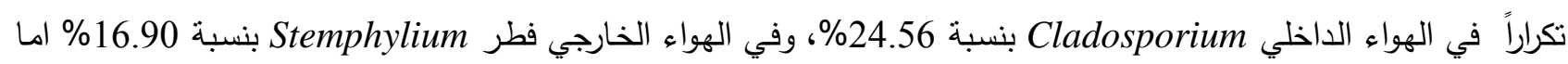
في الموسم الثاني فكان فطر Stemphylium و بنسبة 16.94\% في الهواء الداخلي و فطر Rhizoctoni بنسبة 17.24\% من الهواء الخارجي. كان مجموع وحدات تكوين المستعمرات في الموسم الاول 128، منها 57 في الهواء الداخلي، و 71 في الهواء الخارجي، و في الموسم الثاني بلخ مجموعا 166 منها 118 في الهواء الداخلي و 58 في الهواء الخارجي (الجدول 4). 


\begin{tabular}{|c|c|c|c|c|c|c|c|c|c|c|}
\hline $\begin{array}{c}\% \\
\text { للتكرار }\end{array}$ & الخارجي & $\begin{array}{c}\% \\
\text { للتكرار }\end{array}$ & الداخلي & مستعوين & $\begin{array}{c}\text { \% للتكرار } \\
\text { لل }\end{array}$ & الخارجي & $\begin{array}{c}\text { \% للتكرار } \\
\text { ل }\end{array}$ & الداخلي & مستعوين & الفطريات \\
\hline 19.04 & 8 & 4.92 & 7 & 15 & 12.5 & 1 & 6.25 & 4 & 5 & \\
\hline 0 & 0 & 3.51 & 5 & 5 & 0 & 0 & 3.12 & 2 & 2 & Aspergillus spp \\
\hline 9.52 & 4 & 6.33 & 9 & 13 & 12.5 & 1 & 3.12 & 2 & 3 & Aureobasidium pullulans \\
\hline 7.14 & 3 & 16.90 & 24 & 27 & 25.0 & 2 & 35.93 & 23 & 25 & Cladosporium spp \\
\hline 9.52 & 4 & 2.11 & 3 & 7 & 0 & 0 & 0 & 0 & 0 & Drechslera $\mathrm{spp}$ \\
\hline 0 & 0 & 0.70 & 2 & 2 & 0 & 0 & 0 & 0 & 0 & Fusarium spp \\
\hline 0 & 0 & 0.70 & 1 & 1 & 0 & 0 & 0 & 0 & 0 & Macrophmina phaseolina \\
\hline 0 & 0 & 2.81 & 4 & 4 & 0 & 0 & 0 & 0 & 0 & Nematospora spp \\
\hline 0 & 0 & 0.70 & 1 & 1 & 0 & 0 & 0 & 0 & 0 & Papulaspora equi \\
\hline 19.09 & 8 & 9.85 & 14 & 22 & 12.5 & 1 & 35.93 & 23 & 24 & Penicillium spp \\
\hline 11.90 & 5 & 16.90 & 24 & 29 & 12.5 & 1 & 14.06 & 9 & 10 & Rhizoctoni solani \\
\hline 0 & 0 & 0 & 0 & 0 & 12.5 & 1 & 0 & 0 & 1 & Rhizopus stolonifer \\
\hline 19.09 & 8 & 11.97 & 17 & 25 & 12.5 & 1 & 0 & 0 & 0 & Stemphylium herbarum \\
\hline 0 & 0 & 0.70 & 1 & 1 & 0 & 0 & 0 & 0 & 0 & Trichocladium spp. \\
\hline 0 & 0 & 0.70 & 1 & 1 & 0 & 0 & 0 & 0 & 0 & Trichoderma spp \\
\hline 0 & 0 & 2.11 & 3 & 3 & 0 & 0 & 0 & 0 & 0 & Trichophyto $n \mathrm{ssp}$ \\
\hline 0 & 0 & 0.70 & 1 & 1 & 0 & 0 & 0 & 0 & 0 & Sterile mycelium \\
\hline 4.76 & 2 & 17.60 & 25 & 27 & 0 & 0 & 1.56 & 1 & 1 & Yeast \\
\hline 100 & 42 & 100 & 142 & 184 & 100 & 8 & 100 & 64 & 72 & المجموع \\
\hline
\end{tabular}

وتظهر نتائج (الجدول 5) عزل اَحََ عَثَرَ جنسا من الفطريات فضلا عن الفطريات العقيمة و الخمائر في الموسم الأول، و كان أكثرها تكراراً الفطر Cladosporium وبنسبة 43.75\% في الهواء الداخلي و فطر Penicillium بنسبة 20.83\% في الهواء الخارجي. اما في الموسم الثاني فكان فطر Cladosporium أكثر نكرار في الهواء الداخلي و بنسبة 34.9\%، و فوراء فطر Alternaria هواء داخلي و 24 هواء خارجي و في الموسم الثاني 146 منها 106 هواء داخلي و 40 هواء خارجي.

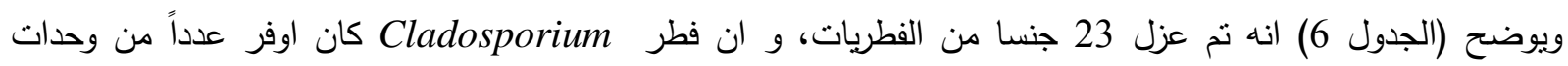
تكوين المستعرات، إذ وصل إلى 99 في الموسم الأول، يليه فطر Penicillium (58)، و في الموسم الثاني أيضا كان فطر Cladosporium أكثر الفطريات المعزولة إذ وصل عددها الى 124 يليه فطر Alternaria (104).

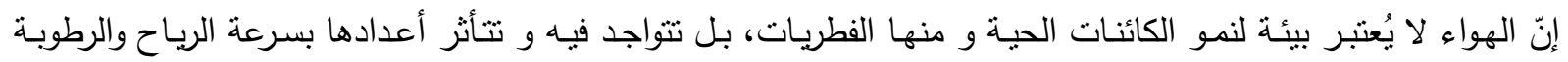

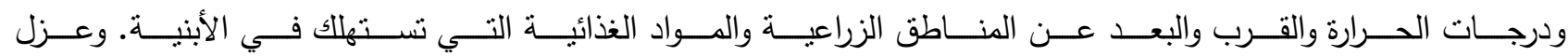

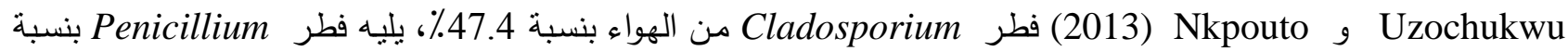

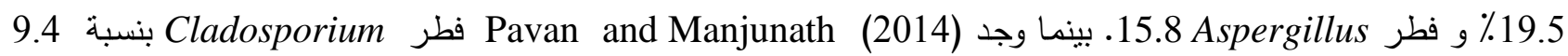

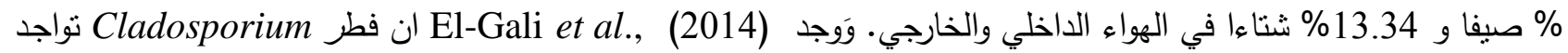

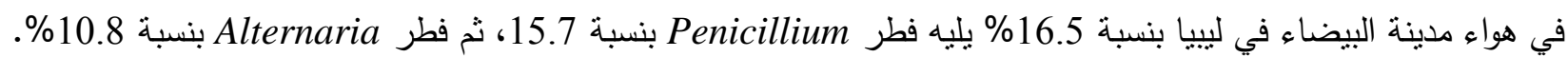




\begin{tabular}{|c|c|c|c|c|c|c|c|c|c|c|}
\hline \multicolumn{5}{|c|}{ الموسم الثاني } & \multicolumn{5}{|c|}{ الموسم الاول } & \multirow[t]{4}{*}{ الفطريات } \\
\hline$\%$ & الهواء & $\%$ & الهواء & وحدة ا & $\%$ & الهواء & $\%$ & الهواء & 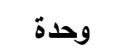 & \\
\hline ل ل ل لتكرار & الخارجي & 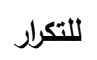 & الداخلي & ت تكوين & ل لتكرار & الخارجي & ل لتكرار & الاخلي & تكوين & \\
\hline & & & & مستعمرة & & & & & مستعمرة & \\
\hline 15.51 & 9 & 11.01 & 13 & 22 & 12.67 & 9 & 10.52 & 6 & 15 & Alternaria alternata \\
\hline 1.72 & 1 & 6.77 & 8 & 9 & 11.26 & 8 & 7.01 & 4 & 12 & Aspergillus spp \\
\hline 10.34 & 6 & 16.10 & 19 & 25 & 8.45 & 6 & 8.77 & 5 & 11 & Aureobasidium pullulans \\
\hline 1.72 & 1 & 0 & 0 & 1 & 0 & 0 & 0 & 0 & 0 & Chetomium spp. \\
\hline 10.34 & 6 & 11.86 & 14 & 20 & 5.63 & 4 & 24.56 & 14 & 18 & Cladosporium spp \\
\hline 1.72 & 1 & 0 & 0 & 1 & 0 & 0 & 0 & 0 & 0 & Curvularia spp. \\
\hline 6.89 & 4 & 7.62 & 9 & 13 & 5.63 & 4 & 0 & 0 & 4 & Drechslera spp \\
\hline 5.17 & 3 & 0 & 0 & 3 & 0 & 0 & 0 & 0 & 0 & Fusarium spp. \\
\hline 0 & 0 & 0.84 & 1 & 1 & 0 & 0 & 0 & 0 & 0 & Helmenthosporium spp. \\
\hline 0 & 0 & 0.84 & 1 & 1 & 1.40 & 1 & 0 & 0 & 1 & Macrophmina phaseolina \\
\hline 0 & 0 & 0 & 0 & 0 & 1.40 & 1 & 0 & 0 & 1 & Nematospora spp \\
\hline 8.62 & 5 & 9.32 & 11 & 16 & 14.08 & 10 & 12.28 & 7 & 17 & Pencillium spp \\
\hline 17.24 & 10 & 13.55 & 16 & 26 & 8.45 & 6 & 14.03 & 8 & 14 & Rhizoctoni solani \\
\hline 5.17 & 3 & 16.94 & 20 & 23 & 16.90 & 12 & 7.01 & 4 & 16 & Stemphylium herbarum \\
\hline 1.72 & 1 & 4.23 & 5 & 6 & 2.81 & 2 & 1.75 & 1 & 3 & Trichphyton spp \\
\hline 3.44 & 2 & 0 & 0 & 2 & 1.40 & 1 & 1.75 & 1 & 2 & Uloclodium spp \\
\hline 0 & 0 & 0.84 & 1 & 1 & 7.03 & 5 & 7.01 & 4 & 9 & Sterile mycelium - \\
\hline 10.34 & 6 & 0 & 0 & 6 & 2.81 & 2 & 5.26 & 3 & 5 & Yeast \\
\hline 100 & 58 & 100 & 118 & 166 & 100 & 71 & 100 & 57 & 128 & المجموع \\
\hline
\end{tabular}

الجدول 5: الفطريات المعزولة من الهواء الداخلي والخارجي للمدارس الثانوية للبنات في مدينة تكريت

\begin{tabular}{|c|c|c|c|c|c|c|c|c|c|c|}
\hline \multicolumn{5}{|c|}{ الموسم الثاني } & \multicolumn{5}{|c|}{ الموسم الاول } & \multirow[t]{3}{*}{ الفطريات } \\
\hline$\%$ & الهواء & $\%$ & الهواء & وحدة & $\%$ & الهواء & $\%$ & الهواء & وحدة & \\
\hline للتكرار & الخارجي & للتكرار & الداخلي & مستعمرة & للتكرار & الخارجي & للتكرار & الداخلي & مستعمرة & \\
\hline 37.5 & 15 & 17.92 & 19 & 34 & 4.16 & 1 & 6.25 & 2 & 3 & Alternaria alternata \\
\hline 7.5 & 3 & 2.83 & 3 & 6 & 16.66 & 4 & 9.37 & 3 & 7 & Aspergillus spp \\
\hline 12.5 & 5 & 4.71 & 5 & 10 & 4.16 & 1 & 3.12 & 1 & 2 & Aureobasidium pullulans \\
\hline 0 & 0 & 0.94 & 1 & 1 & 0 & 0 & 0 & 0 & 0 & Chetomium \\
\hline 15 & 6 & 34.90 & 37 & 43 & 16.66 & 4 & 43.75 & 14 & 18 & Cladosporium spp \\
\hline 5 & 2 & 1.88 & 2 & 4 & 0 & 0 & 0 & 0 & 0 & Fusarium spp. \\
\hline 0 & 0 & 0 & 0 & 0 & 0 & 0 & 3.12 & 1 & 1 & Mycosporium spp \\
\hline 0 & 0 & 0 & 0 & 0 & 0 & 0 & 3.12 & 1 & 1 & Nematospora spp \\
\hline 5 & 2 & 16.98 & 18 & 20 & 20.83 & 5 & 6.25 & 2 & 7 & Pencillium spp \\
\hline 7.5 & 3 & 8.49 & 9 & 12 & 4.16 & 1 & 6.25 & 2 & 3 & Rhizoctoni solani \\
\hline 2.5 & 1 & 7.54 & 8 & 9 & 4.16 & 1 & 3.12 & 1 & 2 & Stemphylium herbarum \\
\hline 0 & 0 & 0.94 & 1 & 1 & 0 & 0 & 3.12 & 1 & 1 & Trichphyton spp \\
\hline 2.5 & 1 & 0.94 & 1 & 2 & 0 & 0 & 0 & 0 & 0 & Uloclodium spp \\
\hline 0 & 0 & 0 & 0 & 0 & 4.16 & 1 & 6.25 & 2 & 3 & Sterile mycelium \\
\hline 5.0 & 2 & 1.88 & 2 & 4 & 25 & 6 & 6.25 & 2 & 8 & Yeast \\
\hline 100 & 40 & 100 & 106 & 146 & 100 & 24 & 100 & 32 & 56 & 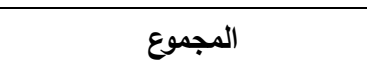 \\
\hline
\end{tabular}


ويوضح (الجدول 6) انه تم عزل 23 جنسا من الفطريات، و ان فطر Cladosporium أعطى أعلى عدداً من وحدات تكوين المستعمرات و قد وصل إلى 99 في الموسم الأول، يليه فطر

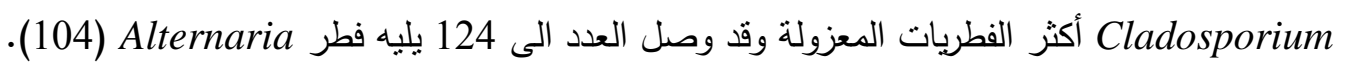
الهواء لا يعتبر بيئة لنمو الكائنات الحية ومنها الفطريات بل تتواجد فيه وتتأثز أعدادها بسرعة الرياح والرطوبة ولاتية ودرجات

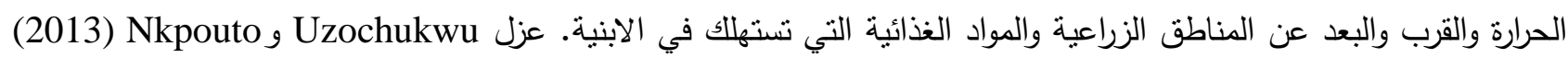

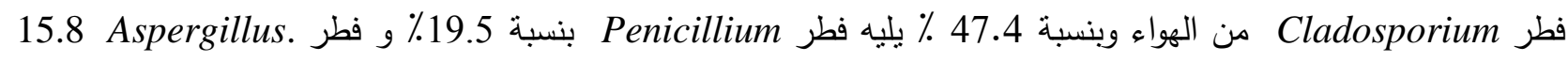

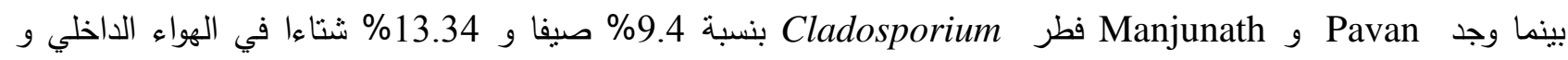

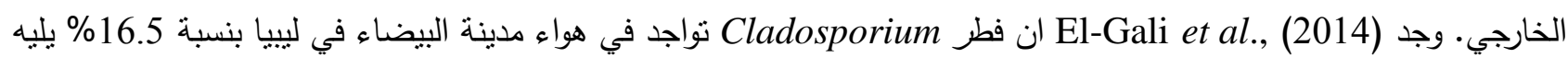
فطر Penicillium و بنسبة 15.7 ثم فطر Alternaria بنسبة 10.8\%.

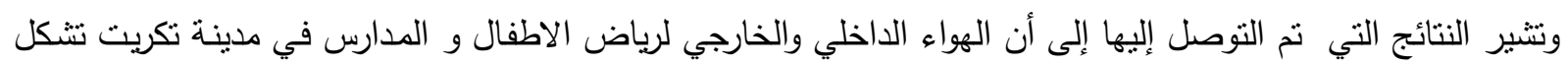

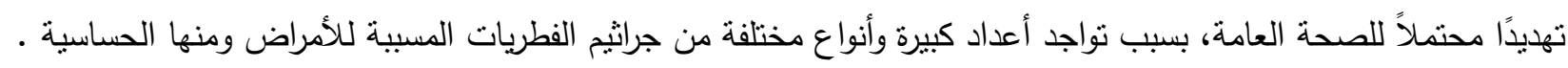
الجدول 6: أعداد وحدات تكوين المستعمرات للفطريات المعزولة من هواء مدارس مدينة تكريت

\begin{tabular}{|c|c|c|c|}
\hline \multicolumn{2}{|c|}{ أعداد تكوين وحدات المستعمرات } & \multirow[t]{2}{*}{ الفطريات } & \multirow[t]{2}{*}{$ت$} \\
\hline الموسم الثاني & الموسم الاول & & \\
\hline 104 & 32 & Alternaria & 1 \\
\hline 0 & 1 & Ascochyta & 2 \\
\hline 24 & 26 & Aspergillus & 3 \\
\hline 64 & 22 & Aurobasidium & 4 \\
\hline 2 & 0 & Chetomium & 5 \\
\hline 124 & 99 & Cladosporium & 6 \\
\hline 13 & 4 & Curvularia & 7 \\
\hline 23 & 7 & Drechlera & 8 \\
\hline 11 & 3 & Fusarium & 9 \\
\hline 2 & 0 & Helminthspoium & 10 \\
\hline 3 & 1 & Macrophomina & 11 \\
\hline 2 & 9 & Mucor & 12 \\
\hline 4 & 2 & Nematosporium & 13 \\
\hline 1 & 0 & Papulaspora & 14 \\
\hline 81 & 58 & Penicillium & 15 \\
\hline 85 & 34 & Rhizoctomia & 17 \\
\hline 0 & 2 & Rhizopus & 18 \\
\hline 75 & 23 & Stemphylium & 19 \\
\hline 1 & 0 & Trichcladium & 20 \\
\hline 2 & 0 & Trichoderma & 21 \\
\hline 10 & 5 & Trichphyton & 22 \\
\hline 4 & 3 & Ulocladium & 23 \\
\hline 2 & 18 & Steril mycelium & 24 \\
\hline 48 & 23 & Yeast & 25 \\
\hline
\end{tabular}

\section{المصادر العربية}

الثرقاوي، حسن عبد الله (2010). دور الفطريات في الصناعة. المعهد القومي لعلوم البحار والمصايد فرع الإسكندرية، مصر. 


$$
\begin{aligned}
& \text { مشاط، بسام بن حسين بن حسن (2013). هل التعرض لفطريات الأماكن المغلقة يسبب أمراض خاصة. قسم البحوث البيئية }
\end{aligned}
$$

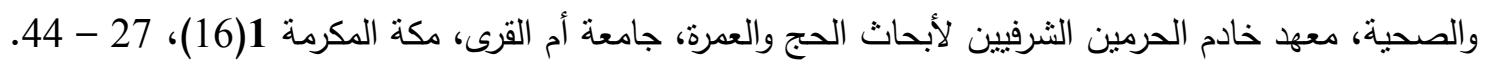

$$
\begin{aligned}
& \text { نخيلان، عبد العزيز مجيد (2011). الفطريات الطبية. عمان. دار دجلة. } \\
& \text { المصادر الأجنبية }
\end{aligned}
$$

Basilico, M.; Chiericatti, C.; Aringoli, E.E.; Althaus, R.L.; Basilico, J.C. (2007). Influence of environmental factors on airborne fungi in houses of Santa Fe City, Argentina. Sci. The Total Enviro., 376(1-3), 143-150

Charles, B.; Freddy, P.; Minati, D.; Jay, P. (2009). Alternaria and Cladosporium fungal allergen epitopes are denatured by sodium hypochlorite. World Allergy Organ J. 2(12), 296-302

de Hoog, G.S.; Guarro, J. (1995). "Atlas of Clinical Fungi". Centraalbureau voor schimmelcultures, Netherlands. 720 p.

Denning, D.W.; Driscoll, B.R.; Hogaboam, C.M.; Bowyer, P.; Niven, R.M. (2006). The link between fungi and sever asthma a summary of evidence. Europ. Respir. J., 27(3), 615-626.

El-Gali, Z.I.; Abdullrahman E.M.; Obeady, N.A. (2014). Isolation and identification of airborne fungi in Indoor/ Outdoor of Home in El-Beida City (Libya). I. J. A. Res. 2(5), 835-843.

Ellis, M.B. (1971). "Dematiaceous Hyphomycetes". Common Wealth Mycological Institute. Kew, Surrey. England. 608 p .

Hedayati, M.; Mayahi, S.; Aghili, R.; Goharimoghadam, K. (2005). Airborne fungi in indoor and outdoor of asthmatic patients home, living in the city of Sari. Iranian J. Allerg. Asthma and Immunol. 4(4), 189-191.

Jawetz, E.; Melnick, J.L.; Adelberg, E.A.; Brooks, G.F.; Butel, J.S.; Morse, S.A. (1998). "Medical Microbiology". $21^{\text {st }}$ ed. Middle east ed. Appleton and Lange. Norwalk, connect: cut/Los Altos, California.

Lehrer, S.B.; Aukrust, L.; Salvaggio, J.E. (2013). Respirotory allergy induced by fungi. Clinics in Medicine, 4, 23-41.

Pavan, R.; Manjunath, K. (2014). Indoor study on airborne fungi in swine house of Bangalore, India. Int. J. Curr. Sci., 9, 77-82

Pitt, J.I.; Hocking, A.D. (1997). "Fungi and Food Spoilage". $2^{\text {nd }}$ ed. Gaithersburg, Maryland: Chapman and Hall. 593 p.

Shelton, B.G.; Kirkland, K.H.; Flanders, W.D.; Morris, G.K. (2002). Profiles of airborne fungi in buildings and outdoor environments in the United States. Applied and Environmental Microbiology, 68(4), 1743 - 1753.

Torres-Rodríguez, J.; Pulido-Marrero, Z.; Vera-García, Y. (2012). Respiratory allergy to fungi in Barcelona, Spani: Clinical aspects, diagnosis and specific treatment ina general allergy unti. Allergologia et Immunopathologia. 40(5), 295 - 300.

Uzochukwu, O.V.; Nkpouto, N. (2013). Airborne fungi in the indoor and outdoor environments of a higher institution in Nigeria. I.J.A.B.R., 3(1), 2013, 9-12.

Watanabe, T.(2002). "Pictorial Atlas of Soil and Seed Fungi - Morphologies of Cultured Fungi and Key to Species". $2^{\text {nd }}$ ed. Boca Ratón: CRC Press. 